Document downloaded from:

http://hdl.handle.net/10251/38255

This paper must be cited as:

Sánchez-Vega, F.; Villatoro-Tello, E.; Montes-Y-Gómez, M.; Villaseñor-Pineda; Luis; Rosso, P. (2013). Determining and Characterizing the Reused Text for Plagiarism Detection. Expert Systems with Applications. 40(5):1804-1813. doi:10.1016/j.eswa.2012.09.021.

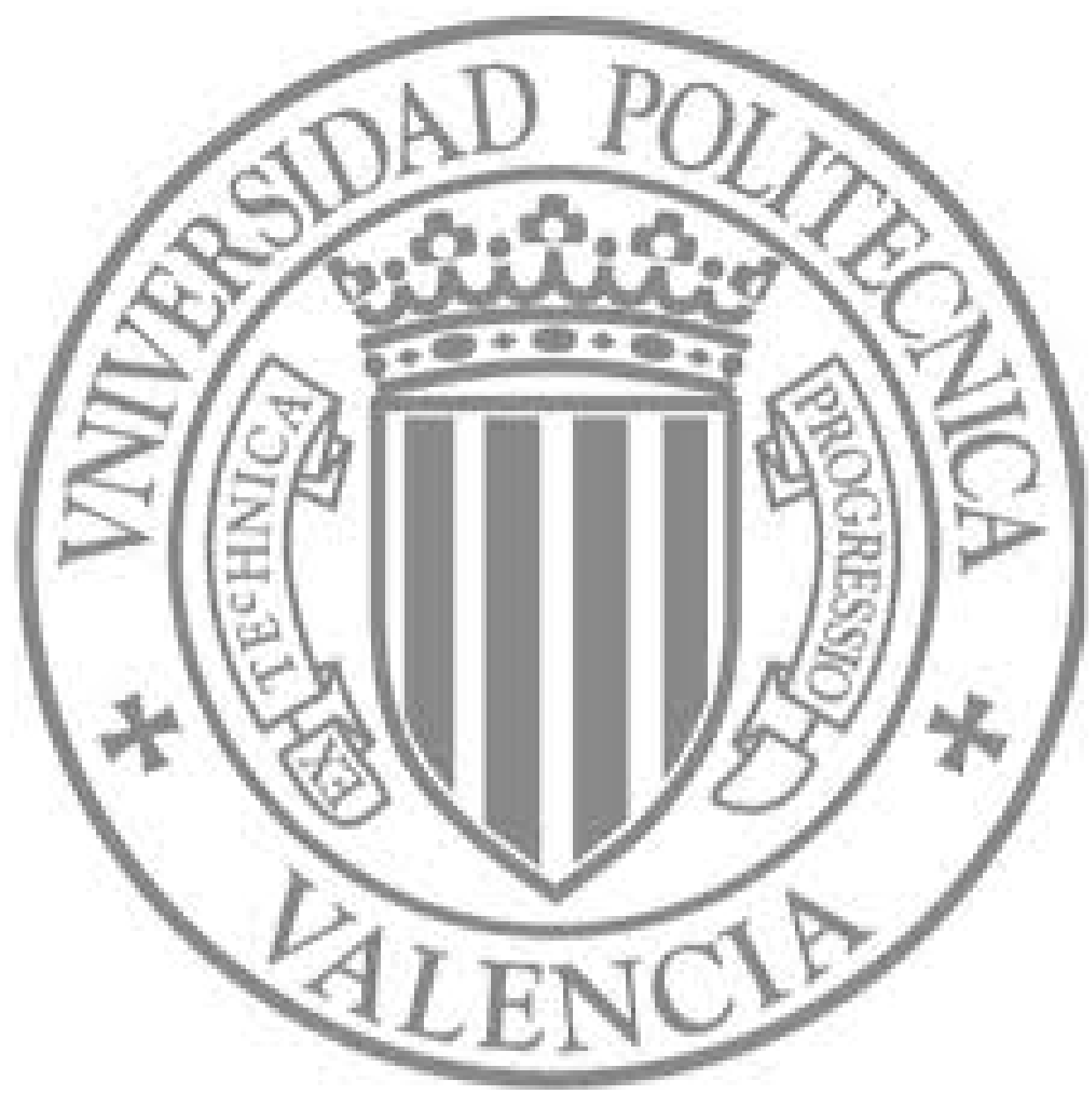

The final publication is available at

http://dx.doi.org/10.1016/j.eswa.2012.09.021

Copyright Elsevier 


\title{
Determining and Characterizing the Reused Text for Plagiarism Detection ${ }^{\text {光 }}$
}

\author{
Fernando Sánchez-Vega ${ }^{\mathrm{a}, *}$, Esaú Villatoro-Tello ${ }^{\mathrm{a}, * *}$, Manuel Montes-y-Gómez ${ }^{\mathrm{a}, * *}$, \\ Luis Villaseñor-Pineda ${ }^{\mathrm{a}}$, Paolo Rosso ${ }^{\mathrm{b}}$ \\ ${ }^{a}$ Laboratory of Language Technologies, Department of Computational Sciences, \\ National Institute of Astrophysics, Optics and Electronics (INAOE), Mexico \\ ${ }^{b}$ Natural Language Engineering Lab., ELiRF \\ Universidad Politecnica de Valencia, Spain.
}

\begin{abstract}
An important task in plagiarism detection is determining and measuring similar text portions between a given pair of documents. One of the main difficulties of this task resides on the fact that reused text is commonly modified with the aim of covering or camouflaging the plagiarism. Another difficulty is that not all similar text fragments are examples of plagiarism, since thematic coincidences also tend to produce portions of similar text. In order to tackle these problems, we propose a novel method for detecting likely portions of reused text. This method is able to detect common actions performed by plagiarists such as word deletion, insertion and transposition, allowing to obtain plausible portions of reused text. We also propose representing the identified reused text by means of a set of features that denote its degree of plagiarism, relevance and fragmentation. This new representation aims to facilitate the recognition of plagiarism by considering diverse characteristics of the reused text during the classification phase. Experimental results employing a supervised classification strategy showed that the proposed method is able to outperform traditionally used approaches.
\end{abstract}

\footnotetext{
This work was done under partial support of CONACyT project grants: (XXXX), and scholarships: (XXXX)

* Principal corresponding author

** Corresponding author

Email addresses: fer.callot l @inaoep.mx (Fernando Sánchez-Vega), villatoroe@inaoep.mx (Esaú Villatoro-Tello), mmontesg@inaoep.mx (Manuel Montes-y-Gómez), villasen@inaoep.mx (Luis Villaseñor-Pineda), prosso@dsic.upv.es (Paolo Rosso)
} 
Keywords: Plagiarism detection, Text reuse, Machine learning, supervised classification

\section{Introduction}

Plagiarism is known as intellectual theft: it consists in using words (ideas) of others and presenting them as your own. Nowadays, due to current technologies for creating and disseminating electronic information, it is very simple to compose a new document by copying sections from different sources extracted from the Web. This situation has caused the growing of the plagiarism phenomenon, and, at the same time, it has motivated the development of tools for its automatic detection.

Very recently, major publishers, namely Elsevier and Springer have showed their interest and concern to fight plagiarism [5]. Hence, by using a software called CrossCheck, they scan submitted papers with the aim of finding verbatim or almost identical chunks of text that already appear in previously published papers. Several tests using the CrossCheck software over different journals showed that from $6 \%$ to $23 \%$ of the submitted articles had to be rejected because they contain a considerable degree of plagiarism. Although CrossCheck is able to uncover plagiarists, the software is susceptible to find false positives, since it only estimates a percentage of similarity between documents.

In this paper we focus on the problem of discriminating plagiarized from freeplagiarized suspicious documents by determining the reused text sections from an original document. We assume that plagiarism is done by reusing some portions of text that can not be considered as common knowledge of the domain. In particular, we consider the task of finding similarities between a suspicious document and a given original document that are more than just a coincidence and more likely to be result of copying [8]. This is a very complex task since reused text is commonly modified with the aim of covering or camouflaging the plagiarism. To date, most approaches have only partially addressed this issue by measuring lexical and structural similarity of documents by means of different kinds of features such as single words [9, 27], fixed length substrings (i.e., $n$-grams) [3, 9], variable length substrings $[4,9]$, dependency relations or a combination of them [7]. The main drawback of these approaches is that they carry out the classification considering only information about the degree of overlap between the suspicious and source documents. Therefore, these strategies are affected by the thematic correspondence of the documents, which implies the existence of common domain-specific 
word sequences, and, as consequence causes an overestimation of their overlap [8].

In order to tackle the above problem we propose a novel approach for finding the portions of possible reused text. Our method, called the Rewriting Index, assigns a weight to each word contained in the suspicious document that describes its degree of membership to a possible portion of plagiarized text. This way, the proposed method is able to discover text that has suffered from some modifications such as word elimination, insertion, and transposition, allowing to perform a partial matching between documents (i. e. find portions of text that are similar but with some change by a paraphrasing). Additionally, we also consider more information during the classification process of the documents. Our idea is to characterize the portions of possible reused text by their relevance and fragmentation. In particular, we consider a set of features that denote the frequency of occurrence of portions of reused text as well as their length distribution. Our hypothesis is that the larger and the less frequent the portions of reused text, the greater the evidence of plagiarism. In other words, we consider that frequent portions of reused text tend to correspond to domain specific terminology, and that small portions of possible reused text may be co-incidental, and therefore, they are not a clear signal of plagiarism.

The experimental evaluation of the proposed approach was carried out on a subset of the METER corpus [12] and on the Plagiarised Short Answers corpus [10]. In particular, we model the document plagiarism detection as a classification problem. Our goal was to show that using the portions of reused text obtained with the Rewriting Index method, and characterizing them by the proposed set of features, it is possible to achieve a greater discrimination performance between plagiarized and non-plagiarized documents than only considering their general degree of overlap.

The rest of the paper is organized as follows. Section 2 presents some recent work on plagiarism detection. Section 3 describes the proposed algorithm for finding portions of possible reused text as well as the formal definition of the proposed features. Section 4 presents the experimental configuration as well as the results achieved in the two test collections. Finally, Section 5 depicts our conclusions and formulates some directions for future work.

\section{Related Work}

One of the main tasks in plagiarism detection consists in determining if the similarities between a suspicious and a source (original) document are more than 
just coincidence and more likely to be result of copying [8]. Broadly speaking, this task includes two main phases: the searching of plagiarism evidence, and the classification of plagiarized documents based on the accumulated evidence.

The main purpose of the first phase is to find similar or reused text portions between the given two documents. Some works have searched for these similarities at the syntactic level by identifying common POS sequences $[16,7]$. On the other extreme, some works have searched for similarities at the lexical level, using common single words as the main evidence of plagiarism [27, 24, 17]. Finally, in between these two approaches, there are works that consider word sequences. Some of them search for common fixed-length sequences known as $n$-grams $[22,13,6,3,2,19,21,14]$, whereas others have used variable length sequences in order to preserve the integrity of the evidence $[6,4,9,18]$.

In the second phase the collected evidence is transformed on a measure or set of measures that indicate the level of copy in the suspicious document. Particularly, most current methods use a representation based on the proportion of positive evidence in relation to the size of the suspicious document $[19,22,15,21,14]$ or to the size of both documents $[6,25,1]$. This representation is used in the documents classification process; common approach consists of applying a manuallydefined threshold function on the computed measure [26, 4, 23, 3, 21]. On the contrary, when the plagiarism evidence is expressed by a set of measures, most methods apply machine learning techniques to automatically define the threshold function $[16,7,9,11]$.

In this paper we propose some ideas to enhance both phases of the plagiarism detection process. First, we propose a new method to find the portions of possible reused text. This method uses a fuzzy string matching automata that is able to detect common actions of plagiarism such as word deletion, insertion and transposition, and, therefore, that allows to collect evidence with a high degree of rewriting, which current methods tend to ignore. Second, we propose a new representation of the plagiarism evidence that helps to describe more appropriately its relevance and diversity and, consequently, allows taking further advantage of the capabilities of machine learning techniques to handle representations with multiple features.

\section{Proposed Method}

As stated in previous sections, common word sequences between the suspicious and source documents are considered the primary evidence of plagiarism. 
Nevertheless, using their presence as unique indicator of plagiarism could be unreliable, since thematic coincidences also tend to produce sequences of common text (i.e., false positives). In addition, even a minor modification to obfuscate the plagiarism will avoid the identification of the corresponding sequences, generating false negatives.

In order to handle the above problems, we propose a novel strategy for detecting plagiarised text called the Rewriting Index method. This method is able to identify portions of reused text even if they have suffered from some modifications. Additionally, we aim to facilitate the recognition of plagiarism by considering diverse characteristics of the portions of reused text during the classification phase.

In the following section we give a brief description of the Turing machine formalism, which will allow us to better describe, in Section 3.2, our proposed algorithm for identifying and extracting the possible reused text between the suspicious $\left(D^{S}\right)$ and the original document $\left(D^{O}\right)$. Then, in Section 3.3, we introduce the proposed set of features used to characterize the extracted portions of reused text.

\subsection{Turing machine formalism}

In order to explain the proposed method we are going to employ the Turing Machine (TM) notation. Formally a TM is defined as a 7-tuple with the form:

$$
M=\left\langle Q, \Sigma, \Gamma, \delta, q_{0}, B, F\right\rangle
$$

where:

- $Q$ is a finite, non-empty set of states.

- $\Sigma$ is the set of input symbols.

- $\Gamma$ is a finite, non-empty set of the tape alphabet (symbols).

- $\delta$ is the transition function which is defined as: $\delta\left(q_{i}, X\right)=\left(q_{j}, Y, S\right)$; where $q_{i}$ represents the actual state and $X$ is the symbol that the head of the TM is reading, $q_{j}$ is the next state, $Y$ is the symbol that is written in the cell pointed by the head of the TM, and $S$ indicates the direction of the head shift, which could be either $\leftarrow$ (left shift), $\rightarrow$ (right shift) or $\mathrm{N}$ (no shift).

- $q_{0}$ is the initial state. 
- $B$ is the blank symbol.

- $F$ is the set of final or accepting states.

Accordingly, we will employ the string $X_{1} X_{2} \ldots X_{i-1} q X_{i} X_{i+1} \ldots X_{n}$ to refer at the configuration where:

- $q$ is the actual state of the TM.

- $X_{i}$, the $i$-th symbol from the left, is the symbol pointed by the head of the tape.

- $X_{1} X_{2} \ldots X_{n}$ is the portion of the tape that is between the most left and most right blank symbols (i.e., $B$ )

Our TM will be capable of reading a null entry (i.e., $\varepsilon$ ). Hence, a transition like $\delta\left(q_{i}, \varepsilon\right)=\left(q_{j}, Y, S\right)$ means that the TM will go from the state $q_{i}$ to state $q_{j}$ by reading $\varepsilon$, indicating to the head of the TM to write $Y$, and shifting in to the $S$ direction ${ }^{1}$.

Furthermore, our TM will handle a stack; i.e., it is a pushdown TM. For our purposes, the main goal of the stack is to function as a counter, hence the alphabet of the stack corresponds to the set of the natural numbers $\mathbb{N}$.

Consequently, the transition function for our pushdown TM is defined as: $\delta\left(q_{i}, X, p\right)=\left(q_{j}, Y, p^{\prime}, S\right)$; where $q_{i}$ is the actual state, $X$ is the symbol that the head of the TM is reading and $p$ is the topmost stack symbol, $q_{j}$ is the next state, $Y$ is the symbol that is written in the cell pointed by the head of the TM, $p^{\prime}$ is the symbol that is pushed to the stack (i.e., pop $p$, replacing it by pushing $p^{\prime}$ ), and $S$ indicates the direction of the head shift.

There might be cases when it is not important to know which symbol is at the top of the stack. For denoting such situations we will use $\lambda$ within the transition function: $\delta\left(q_{i}, X, \lambda\right)=\left(q_{j}, Y, p^{\prime}, S\right)$; indicating the TM to pop the topmost stack symbol and replacing it by pushing $p^{\prime}$.

\subsection{Identifying the reused text}

The proposed Rewriting Index method assigns a weight to each word contained in the suspicious document describing its degree of membership to a possible portion of reused text. Hence, it is able to identify portions of text that although

\footnotetext{
${ }^{1}$ Notice that a null entry $\varepsilon$ is different from the blank symbol $B$.
} 
they do not represent an exact match, they indicate highly probable plagiarized sections. In other words, this method is able to obtain non-consecutive portions of reused text and, therefore, to capture the common actions of a plagiarist such as word elimination, insertion and transposition.

In particular, the proposed method is an ad-hoc search algorithm that uses a context window of size $v$, that contains $v$ words from the original document $D^{O}$ (i.e., our search algorithm moves through the text of $D^{O}$ ). The position of this context window is defined by its middle word, which is, from a Turing machine perspective, the position where the head of the tape is pointing to. We will refer to the word positioned at middle of the context window as the focus.

Therefore, if we take for granted that the tape of our TM are the words contained in $D^{O}$ (i.e., the original document), represented by the string:

$$
w_{1}^{O} w_{2}^{O} \ldots w_{i-1}^{O} q w_{i}^{O} w_{i+1}^{O} \ldots w_{n}^{O}
$$

where the central word of the context window is the $i$-th word, which is the position where the head of the tape is pointing to; being $q$ the actual state of the $\mathrm{TM}^{2}$. Notice that $v$ has to be an odd number in order to have the same number of context words $\left(\frac{v-1}{2}\right)$ at the right and at the left of the focus word ${ }^{3}$.

The Rewriting Index algorithm will assign a $R e I$ value to each word $w_{j}^{S}$ (i.e., the word at position $j$ within the suspicious document $\left.D^{S}\right)$. To compute $\operatorname{ReI}\left(w_{j}^{S}\right)$ we define five different TMs (Figures 1 to 5). Each TM will assign a different $R e I$ value $\left(c_{i}\right)$ depending on: the position in $D^{O}$ of the searched word $w_{j}^{S}$. That is, if the searched word appears at the focus the $R e I$ is equal to $c_{1}$ indicating a verbatim case (Figure 1); if the word appears at the right from focus it takes values $c_{2}$ or $c_{4}$ suggesting a moderate or large number of deletion/insertion operations respectively (Figures 2 and 3); if it appears at the left of the focus it takes values $c_{3}$ or $c_{5}$ signifying a moderate or severe word transposition operation (Figures 4 and 5); finally, if the searched word does not appears in $D^{O}$, its $\operatorname{Re} I$ value is equal to 0 .

We assume that every TM acts over the same tape $\left(w_{i}^{O} \ldots w_{n}^{S}\right)$, and we will considerate only the changes (actions) made by the TM that reaches an accepting state. If more than one TM succeed, we will preserve those changes made from

\footnotetext{
${ }^{2} \mathrm{TM}$ notation assume that the word located at the head of the tape will always be $w_{i}^{O}$, i.e., the focus word.

${ }^{3}$ From here we will refer to the words contained within the context window as local words, and to those outside the context window as global words.
} 
the one that obtains the higher $R e I$ value. In general, the constants $c_{i}$ fulfil the following condition: $c_{1}>c_{2}>c_{3}>c_{4}>c_{5}>0$. The following subsections describe in detail each one of the mentioned cases.

\subsubsection{Capturing verbatim copies}

The following automata (Figure 1) is able to identify sequences of consecutive words that had been literally copied from the original document $D^{O}$. Notice that every time this TM reaches the final state $q_{1}$ the $\operatorname{Re} I\left(w_{j}^{S}\right)$ will get the $c_{1}$ value.

The TM from Figure 1 will reach an accepting state when the searched word $w_{j}^{S}$ is equal to the word located at the focus (i.e., $w_{i}^{O}$, the word pointed by the head of the tape). In this case, the TM leaves the same word on that cell of the tape and shifts one position to the right in order to search for another coincidence.

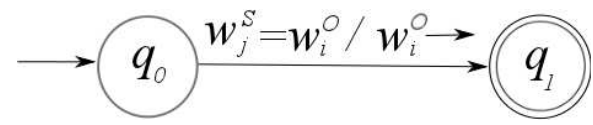

Figure 1: TM capturing a verbatim copying case.

\subsubsection{Capturing deletion/insertion operations}

The TM described in Figure 2 aims to identify moderate cases of word deletion and insertion operations. It is mainly able to identify if a few words, within the local words at the right of the focus, were deleted or inserted. If this situation occurs, the focus is moved to the symbol located after the position where $w_{j}^{S}=w_{i}^{O}$ was accomplished, the $\operatorname{ReI}\left(w_{j}^{S}\right)$ is set to $c_{2}$, and the topmost stack symbol is set to 0 indicating that the position of the focus has changed. As we previously mentioned, our stack works as a counter and we assume that every time the TM is called, the initial stack symbol $p$ is set to 0 . Accordingly, every time the head of the TM is moved, $p$ increases by 1 and the automata verifies if the head continues within the context window, i.e., if $p<\frac{v+1}{2}$.

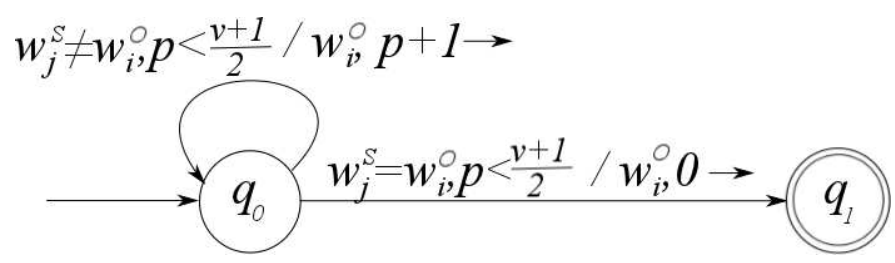

Figure 2: TM capturing a moderate number of deletion and insertion operations. 
There are situations where plagiarists delete or insert a greater number of words between portions of plagiarized text, for such cases we define the TM shown in Figure 3. Notice that the automata defined in this figure will search for $w_{j}^{S}$ among the global words located at the right from the context window. If $w_{j}^{S}$ is found, the TM verifies that the next entry $\left(w_{j+1}^{S}\right)$ also corresponds to a copied word (i.e., verifies if this word is equal to the symbol pointed by the head of the tape), and if that is the case, it reaches the final state $\left(q_{1} \rightarrow q_{3}\right)$, updating the focus word by pushing 0 in the stack, and assigns the value $c_{4}$ to $\operatorname{ReI}\left(w_{j}^{S}\right)$. If the later condition is not accomplished (i.e., $w_{j+1}^{S} \neq w_{i}^{O}$ ), the TM returns the head of the tape to its initial position $\left(q_{1} \rightarrow q_{2} \rightarrow q_{3}\right)$ by using the information provided by the word counter $p$. This step is performed since we consider that finding a single coincidence too far from the context window is not very relevant, but on the contrary, if two coincidences are found it is worth focusing on that section of the document.

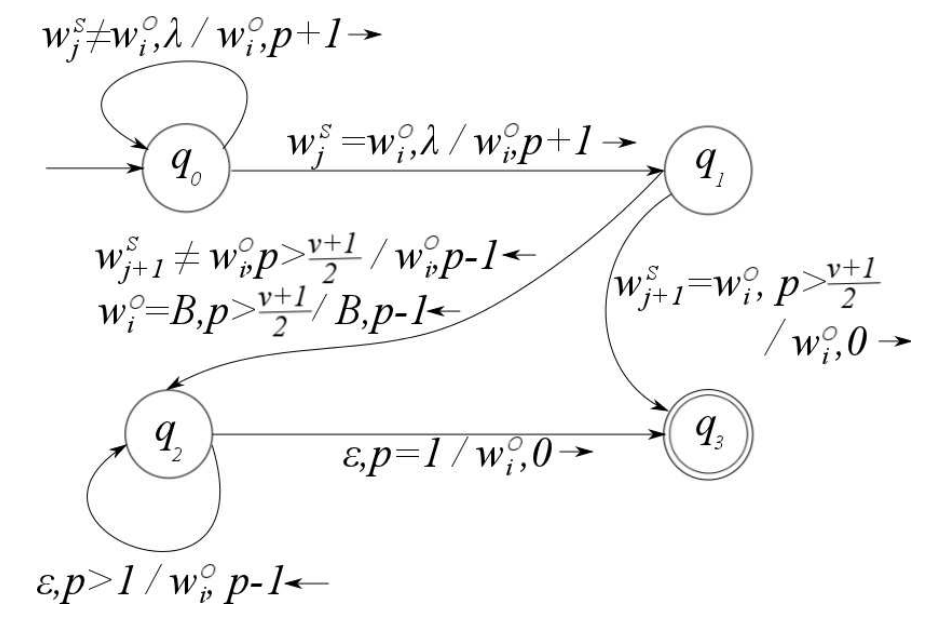

Figure 3: TM capturing a severe number of deletion and insertion operations.

\subsubsection{Capturing word transpositions}

Our method also considers plagiarism cases generated by word transposition operations, where the order of some words has been changed. In particular, the automata shown in Figure 4 searches for $w_{j}^{S}$ within the local words at the left of focus, whereas, the automata in Figure 5 performs the same action but within the global words at the left of the context window. When these automata find the searched word $w_{j}^{S}$, they return the head of the tape to its initial position and assigns 
the value $c_{3}$ to $\operatorname{ReI}\left(w_{j}^{S}\right)$ if the matching occurs within the local words (refer to Figure 4), or a value of $c_{5}$ if it appears in the global words (refer to Figure 5).

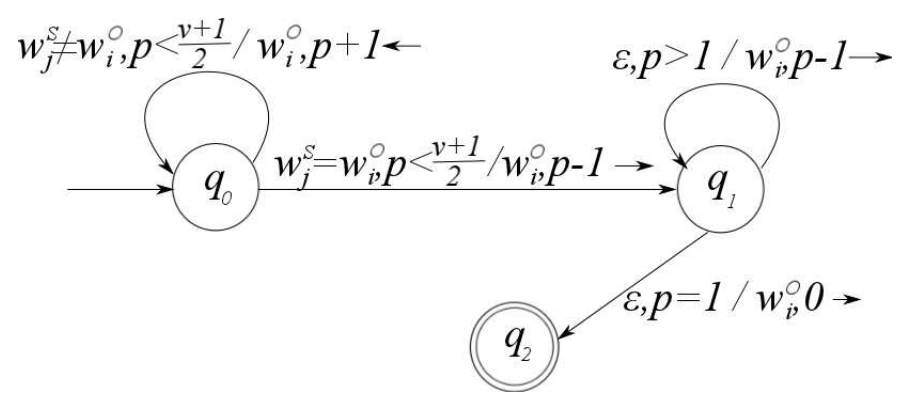

Figure 4: TM capturing a moderate number of word transpositions.

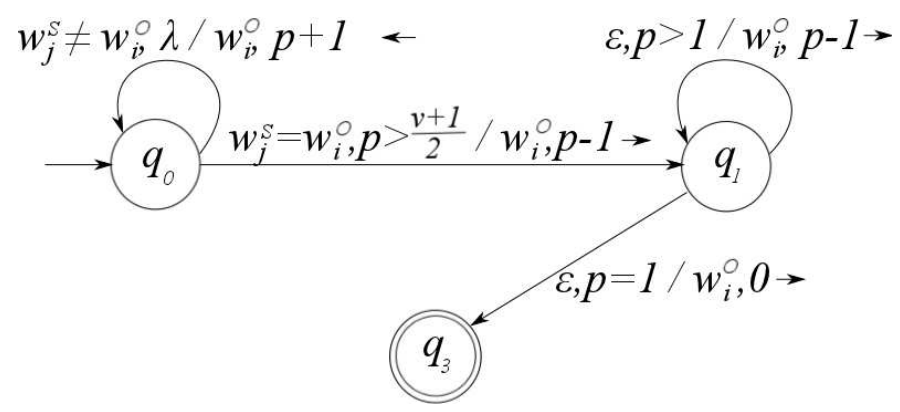

Figure 5: TM capturing a severe number of word transpositions.

\subsubsection{Complexity of the method}

The Rewriting Index algorithm is able to provide a $R e I$ value for each $w_{j}^{S} \in$ $D^{S}$ in a time proportional to $O(m)$ in the best case, being $m$ the number of words contained in $D^{S}$. In this case the suspicious document represents an exact copy of $D^{O}$. The worst case occurs when no word from the suspicious document occurs in the original document, which leads to a time proportional to $O(m n)$ being $n$ the number of words contained in $D^{O}$.

\subsection{Characterizing the reused text}

Once evaluated each word $w_{j}^{S}$ from the suspicious document as described in Section 3.2, we define a portion of reused text as the sequence of consecutive words $p$ denoted by: 


$$
p=\left\langle w_{k}^{S} w_{k+1}^{S} \ldots w_{l-1}^{S} w_{l}^{S}\right\rangle
$$

where $k \leq j \leq l$, and satisfied: $\operatorname{ReI}\left(w_{j}^{S}\right)>c_{4}, \operatorname{Re} I\left(w_{k-1}^{S}\right) \leq c_{4}$ and $\operatorname{Re} I\left(w_{l+1}^{S}\right) \leq c_{4}$, in order to consider only local words inside the portion of possible reused text.

Subsequently, we define $P$ as the set of all the portions of reused text $p$ contained in $D^{S}$. Then, in order to discriminate between plagiarized and nonplagiarized documents, we propose characterizing $P$ by three main types of features, namely the rewriting degree, the relevance and the fragmentation features. The next expression shows the proposed representation of $P$.

$$
\left\langle f^{R e I}, f_{1}^{r l v}, \ldots, f_{m}^{r l v}, f_{1}^{f r g}, \ldots, f_{m^{\prime}}^{f r g}\right\rangle
$$

We represent the set of portions of reused text by $1+m+m^{\prime}$ features, where $f^{R e I}$ represents an agglomerative version of the $R e I$ values computed with our proposed method (Section 3.2), and $f_{i}^{r l v}$ and $f_{j}^{f r g}$ indicate the relevance and the fragmentation of the portions of reused text of length $i$ and $j$ respectively. Cases of particular interest are the $f_{m}^{r l v}$ and $f_{m^{\prime}}^{f r g}$ features which indicate the values of all portions with length equal or greater than $m$ and $m^{\prime}$ words. Their purpose is to deal with the data sparseness and to allow taking advantage of the occurrence of discriminative but very rare longer portions of reused text.

Rewriting degree feature. This feature aims to indicate the degree of plagiarized text contained in the suspicious document $D^{S}$; in other words, it represents how much the words from $D^{S}$ were taken from $D^{O}{ }^{4}$ It is computed as an average of the $R e I$ values from all the words contained in $D^{S}$ as indicated in the following formula:

$$
f^{R e I}=\frac{1}{\left|D^{S}\right|} \sum_{w_{j}^{S} \in D^{S}} \operatorname{ReI}\left(w_{j}^{S}\right)
$$

Fragmentation features. By means of these features we aim to find a relation between the length and quantity of portions of reused text and plagiarism. These features are based on two basic assumptions. On the one hand, we consider that the longer the portions of reused text, the greater the evidence of plagiarism. On

\footnotetext{
${ }^{4}$ This measure not only involves the number of shared words but also if they are in similar contexts.
} 
the other hand, based on the fact that long portions of reused text are very rare, we consider that the more the portions of reused text, the greater the evidence of plagiarism.

According to these basic assumptions we compute the value of the $f_{i}^{f r g}$ feature by adding the lengths of all portions of reused text of length equal to $i$ as described in the following formula:

$$
f_{i}^{f r g}=\sum_{\left\{p_{j}: p_{j} \in P \wedge \text { length }\left(p_{j}\right)=i\right\}} \operatorname{length}\left(p_{j}\right)
$$

The definition of the agglomerative feature $f_{m}^{f r g}$ is stated below:

$$
f_{m}^{f r g}=\sum_{\left\{p_{j}: p_{j} \in P \wedge \operatorname{length}\left(p_{j}\right) \geq m\right\}} \operatorname{length}\left(p_{j}\right)
$$

Relevance features. This second group of features aims to quantify the portions of reused text by their words. That is, they aim to determine the relevance of the portions of reused text with respect to the thematic content of both documents. The idea behind these features is that frequent words or very small portions of reused text are related to the topic of the documents, and not necessarily are a clear signal of plagiarism. On the contrary, they are supported on the intuition that plagiarism is a planned action, and, therefore, that plagiarized sections are not used exhaustively.

In particular we measure the relevance of a given portion of reused text $p_{i} \in P$ by the formula:

$$
\operatorname{rlvc}\left(p_{i}\right)=\prod_{k=1}^{\left|p_{i}\right|} \frac{2}{\operatorname{occ}\left(w_{k}^{p_{i}}, D^{S}\right)+\operatorname{occ}\left(w_{k}^{p_{i}}, D^{O}\right)}
$$

where $\operatorname{occ}\left(w_{k}, D\right)$ indicates the times word $w_{k}$ occurs in $D$.

This measure of relevance castigates the portions of reused text formed by words that are frequent in both documents. The grater value (i.e., rlvc $=1$ ) occurs when the portion of reused text (and all its inner words) appear exclusively once in both documents, indicating that it has a great chance for being a deliberate copy.

Based on the definition of the relevance of a portion of reused text, relevance features are computed as follows:

$$
f_{i}^{r l v}=\sum_{\left\{p_{j}: p_{j} \in P \wedge \operatorname{length}\left(p_{j}\right)=i\right\}} \operatorname{rlvc}\left(p_{j}\right)
$$


The definition of the agglomerative feature $f_{m}^{r e l}$ is as follows:

$$
f_{m}^{r l v}=\sum_{\left\{p_{j}: p_{j} \in P \wedge \operatorname{length}\left(p_{j}\right) \geq m\right\}} \operatorname{rlvc}\left(p_{j}\right)
$$

\section{Experiments and Results}

\subsection{Datasets}

For the experiments we used a subset of the METER corpus [12], a corpus specially designed to evaluate text reuse in the journalism domain. It consists of annotated examples of related newspaper texts collected from the British Press Association (PA) and nine British newspapers that subscribe to the PA newswire service. In particular, we only used the subset of news reports (suspicious documents) that have only one single related note (original document). This subset consists of 253 pairs of documents.

In this corpus each suspicious document (note from a newspaper) is manually annotated with one of three general classes indicating its derivation degree with respect to the corresponding PA news: wholly-derived, partially-derived, and non - derived. For our experiments we considered wholly and partially derived documents as examples of plagiarism and non-derived documents as examples of non-plagiarism, modelling in this way the plagiarism detection task as a two-class classification problem. In particular, the selected subset consists of 181 positive examples of plagiarism and 72 negative cases.

In addition, we also performed experiments using the Plagiarised Short Answers (PSA) corpus [10]. Different to the METER corpus, this collection represents an explicitly-designed corpus of plagiarized documents. In this corpus each suspicious document is annotated with one of four general classes indicating its plagiarism degree with respect to the original document: near - copy, light - revision, heavy - revision and non-plagiarism. For the experiments we considered the four classes, handling the task as a multi-class classification problem. This corpus consists of 95 pairs of documents having the following distribution: 19 near copies, 19 light revisions, 19 heavy revisions and 38 cases of non-plagiarism.

Recently, the PAN-PC corpus ${ }^{5}$ has also been used to evaluate plagiarism detection. This corpus includes plagiarism examples generated by translation and

\footnotetext{
${ }^{5}$ http://pan.webis.de/
} 
automatic methods and is used to evaluate methods that search for reused-text portions from a very large reference collection [20]. Although the relevance of this resource, we decided not to use it because we are mainly interested in modelling and detecting human generated plagiarism.

\subsection{Evaluation}

For the evaluation of the proposed approach, as well as the baseline methods, we employed the Naïve Bayes classification algorithm as implemented by Weka, and applied a 10 times repeated random sub-sampling 10 cross-fold validation strategy. In all cases, we preprocessed the documents by substituting punctuation marks by a generic label, but we did not eliminate stop words nor apply any stemming procedure.

The evaluation of results was carried out mainly by means of the classification accuracy, which indicates the overall percentage of documents correctly classified as plagiarized and non-plagiarized. Additionally, due to the class imbalance, we also present the macro-averaged $F_{1}$ measure as used in [9].

\subsection{On the selection of the parameter values}

As indicated by the Expression 4, we propose representing the portions of reused text in the suspicious document $\left(D^{S}\right)$ by a vector of $1+m+m^{\prime}$ features. In this vector, the first feature indicates the overall degree of plagiarized text, whereas the rest of the features indicate the relevance and fragmentation of the portions of reused text of a particular length, except for the $m$ and $m^{\prime}$-features which integrate information from all portions of reused text with length greater than $m$ and $m^{\prime}$ respectively.

In order to automatically determine an appropriate value of $m$ and $m^{\prime}$, our method, before the classification process; computes the information gain value (IG) of each obtained feature. This automatic process is as follows; given a training set, we extract portions of reused text of lengths varying from 1 to 50 resulting a representation of 101 features. Then, we evaluate the IG score of these features and compute their mean value. Finally, we decided preserving those features having an IG greater than the mean value. Following this procedure our method established for the experiments reported in this paper the following values: for the METER corpus $m=4$ and $m^{\prime}=4$, and for the PSA corpus $m=5$, and $m^{\prime}=1$.

Another important parameter of the proposed method is the size $v$ of the context window. Similar to the definition of $m$ and $m^{\prime}$, we determined the value of $v$ by evaluating the IG of the $f^{R e I}$ feature considering $v$ equal to $9,15,19,25$, and 
29. This process indicated that using a window of size equal to 19 contributes the best for the proposed method in both corpora.

Finally, our method also requires the definition of the constants $c_{i}$, which are the values that each automata assigns when it succeed. For the experiments reported here, these constants were defined as: $c_{i}=1 / i$. Notice that such definition results in the following $c_{i}$ values: $1>\frac{1}{2}>\frac{1}{3}>\frac{1}{4}>\frac{1}{5}>0$. It is also important to notice that these values satisfy the conditions required by the TMs to reach their final states.

\subsection{Results}

\subsubsection{Baseline definition}

As we previously mentioned, most current methods discriminate plagiarized from non-plagiarized documents by evaluating their degree of overlap with the original document using three main kinds of features, namely, single words, fixed length substrings (i.e., ngrams), and variable length substrings. In particular, we generated the baseline results describing the overlap between the suspicious and original documents by means of: (i) the percentage of common words (Baseline 1), and (ii) the percentage of common words extracted from the consecutive common sequences (Baseline 2). It is worth mentioning that both of these techniques are considered hard-baselines.

In addition, for the PSA corpus, we also present the results by Chong et al. [7], which are the best results reported elsewhere for this collection. They measured the overlap between the suspicious and original documents by combining all previous features with information about their common syntactic dependency relations.

\subsubsection{Experiments on the METER corpus}

Table 1 presents the results on the METER corpus. They indicate that the proposed method achieved a higher accuracy and $F_{1}$ measure than the other approaches, outperforming the best baseline configuration (i.e., $1-$ gram) by $5.24 \%$ in terms of accuracy.

Table 1 show that baseline results are very high (above 54\% in terms of accuracy), demonstrating the relevance of the word intersection as main criterion for plagiarism detection. However, notice that our method considering 9 features (ReI, 4- $f^{r l v}$ and 4- $f^{f r g}$ ), which were automatically defined (Section 4.3) is able to perform a better classification process, indicating that there are in fact some actions that single word(s) overlap methods are unable to capture. 


\begin{tabular}{|c|c|c|c|c|}
\hline Method & Features & $\begin{array}{l}\text { Num. of } \\
\text { features }\end{array}$ & Acc. & $F_{1}$ measure \\
\hline Proposed & $f^{R e 1}, f^{r l v}, f^{f r g}$ & 9 & $77.15 \%$ & 0.683 \\
\hline \multirow{10}{*}{ Baseline 1} & 1-grams & 1 & $73.1 \%$ & 0.655 \\
\hline & 2-grams & 1 & $71.1 \%$ & 0.674 \\
\hline & 3-grams & 1 & $66.7 \%$ & 0.644 \\
\hline & 4-grams & 1 & $66.0 \%$ & 0.645 \\
\hline & 5-grams & 1 & $64.0 \%$ & 0.630 \\
\hline & 6-grams & 1 & $62.8 \%$ & 0.620 \\
\hline & 7-grams & 1 & $60.4 \%$ & 0.597 \\
\hline & 8-grams & 1 & $58.1 \%$ & 0.576 \\
\hline & 9-grams & 1 & $56.5 \%$ & 0.563 \\
\hline & 10-grams & 1 & $54.1 \%$ & 0.540 \\
\hline \multirow{10}{*}{ Baseline 2} & CommSeqs(length $\geq 1)$ & 1 & $69.1 \%$ & 0.592 \\
\hline & CommSeqs(length $\geq 2)$ & 1 & $\mathbf{7 2 . 7} \%$ & 0.677 \\
\hline & CommSeqs(length $\geq 3$ ) & 1 & $72.7 \%$ & 0.676 \\
\hline & CommSeqs(length $\geq 4)$ & 1 & $69.1 \%$ & 0.665 \\
\hline & CommSeqs $($ length $\geq 5)$ & 1 & $66.7 \%$ & 0.651 \\
\hline & CommSeqs(length $\geq 6)$ & 1 & $66.7 \%$ & 0.654 \\
\hline & CommSeqs(length $\geq 7$ ) & 1 & $65.6 \%$ & 0.644 \\
\hline & CommSeqs(length $\geq 8)$ & 1 & $63.6 \%$ & 0.627 \\
\hline & CommSeqs $($ length $\geq 9)$ & 1 & $62.4 \%$ & 0.616 \\
\hline & CommSeqs (length $\geq 10)$ & 1 & $60.0 \%$ & 0.593 \\
\hline
\end{tabular}

Table 1: Comparison of the proposed method against baseline approaches on the METER corpus

\subsubsection{Experiments on the PSA corpus}

Similar to the previous section, Table 2 compares the results from our method against defined baselines, including, in this case, the the best result reported in [7]. These results indicate that the proposed method clearly outperformed the best reported configuration (Chong) in accuracy and $F_{1}$ measure by $7.1 \%$ and $8.7 \%$ respectively.

It is important to notice that the best baseline configurations obtained in this experiment were very different from those generated with the METER corpus. These variations took place because of the different characteristics of the two datasets (Section 4.1); they mainly consisted in a better evaluation when the similarity between the suspicious and original documents is obtained using larger n-grams and common sequences.

In addition, Table 3 show obtained performance by our method when different subsets of the proposed TMs are employed during the plagiarism detection task. As it is possible to observe, using only the TM that identifies verbatim sequences allows to correctly classified the near copy and non-plagiarism cases, however the heavy revision class is commonly confused as non-plagiarism. Accordingly, using only the TM that detects transposition actions did not show an 


\begin{tabular}{|c|c|c|c|c|}
\hline Method & Features & $\begin{array}{l}\text { Num. of } \\
\text { features }\end{array}$ & Acc. & $\overline{F_{1} \text { measure }}$ \\
\hline Proposed & $f^{R e 1}, f^{r l v}, f^{f r g}$ & 7 & $75.89 \%$ & 0.701 \\
\hline \multirow{10}{*}{ Baseline 1} & 1-grams & 1 & $61.0 \%$ & 0.516 \\
\hline & 2-grams & 1 & $65.2 \%$ & 0.572 \\
\hline & 3-grams & 1 & $66.3 \%$ & 0.589 \\
\hline & 4-grams & 1 & $65.2 \%$ & 0.577 \\
\hline & 5-grams & 1 & $67.3 \%$ & 0.597 \\
\hline & 6-grams & 1 & $67.3 \%$ & 0.585 \\
\hline & 7-grams & 1 & $65,2 \%$ & 0.569 \\
\hline & 8-grams & 1 & $66.3 \%$ & 0.562 \\
\hline & 9-grams & 1 & $63.1 \%$ & 0.517 \\
\hline & 10-grams & 1 & $62.1 \%$ & 0.492 \\
\hline \multirow{10}{*}{ Baseline 2} & CommSeqs(length $\geq 1)$ & 1 & $62.1 \%$ & 0.522 \\
\hline & CommSeqs (length $\geq 2$ ) & 1 & $63.1 \%$ & 0.540 \\
\hline & CommSeqs (length $\geq 3$ ) & 1 & $65.2 \%$ & 0.574 \\
\hline & CommSeqs (length $\geq 4)$ & 1 & $63.1 \%$ & 0.545 \\
\hline & CommSeqs (length $\geq 5)$ & 1 & $64.2 \%$ & 0.566 \\
\hline & CommSeqs (length $\geq 6$ ) & 1 & $67.3 \%$ & 0.596 \\
\hline & CommSeqs(length $\geq 7)$ & 1 & $68.4 \%$ & 0.603 \\
\hline & CommSeqs (length $\geq 8)$ & 1 & $69.4 \%$ & 0.614 \\
\hline & CommSeqs(length $\geq 9)$ & 1 & $68.4 \%$ & 0.599 \\
\hline & CommSeqs(length $\geq 10)$ & 1 & $65.2 \%$ & 0.556 \\
\hline Chong & Combination $^{6}$ & 7 & $70.53 \%$ & 0.640 \\
\hline
\end{tabular}

Table 2: Comparison of the proposed method against baseline approaches on the PSA corpus

important improvement, nonetheless this automaton it detects more accurately the heavy revision cases than the verbatim automaton. Finally, the automaton that detects deletion/insertion actions showed to be the more accurate across all the plagiarism classes. Nevertheless, using all the TM's results in better performance, particularly for the paraphrase cases (i.e., light and heavy revision) that are the most difficult to detect even for the state-of-the-art methods [7].

\subsection{Further analysis}

As we mentioned in Section 4.3 our method depends on the definition of three main parameters, namely, $m$ which is the length of the relevance features, $m^{\prime}$ that corresponds to the length of the fragmentation features and finally, $v$ that represents the size of the context window. In the following sections we present an analysis of our proposed method when these parameters are manually defined for

\footnotetext{
${ }^{6}$ Chong [7] used the following seven features that combine information at lexical and syntactic level: Trigram Containment Measure (as baseline), Baseline + Lem, Baseline + Stop + Pun + Num, LM - Bigram Perplexity, LM - Trigram Perplexity, Longest Common Subsequence and Dependency Relations.
} 


\begin{tabular}{|c|c|c|c|c|}
\hline \multirow{3}{*}{$\begin{array}{l}\text { Captured } \\
\text { rewriting } \\
\text { actions }\end{array}$} & \multicolumn{4}{|c|}{$F_{1}$ measure } \\
\hline & non & heavy & light & near \\
\hline & plagiarism & revision & revision & copy \\
\hline Verbatim & 0.718 & 0.008 & 0.294 & 0.617 \\
\hline Transpositions & 0.730 & 0.160 & 0.201 & 0.594 \\
\hline Deletion/Insertion & 0.763 & 0.285 & 0.352 & 0.705 \\
\hline All actions & 0.952 & 0.639 & 0.483 & 0.729 \\
\hline Chong & 0.925 & 0.564 & 0.486 & 0.588 \\
\hline
\end{tabular}

Table 3: Performance comparison of the different TM's capturing different rewriting actions

both the METER and the PSA corpus.

\subsubsection{Additional experiments on the METER corpus}

As we mentioned in Section 4.3, the process that automatically selects the parameter values in the METER corpus established that $m=4, m^{\prime}=4$ and $v=19$, allowing our method to achieve a $F_{1}$ score of 0.683 .

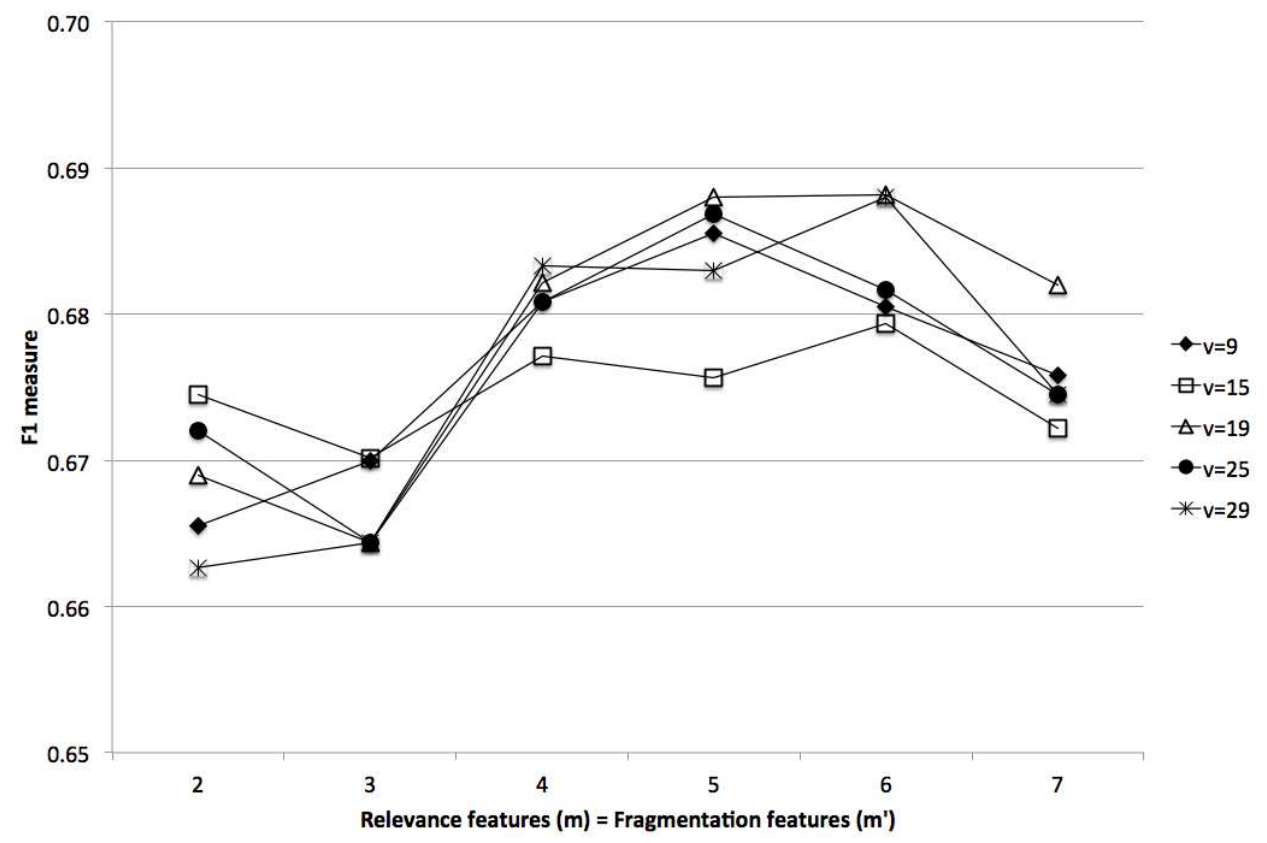

Figure 6: Behaviour of the proposed method when varying the size of the context window $v$, and the maximum length of the relevance and fragmentation features $m$ and $m^{\prime}$ for the METER corpus.

Accordingly, figure 6 depicts the performance of the proposed method, in terms of the $F_{1}$ measure, when varying the size of the relevance and fragmen- 
tation features (i.e., $m$ and $m^{\prime}$ ) as well as the size of the context window $v$. Notice that for these experiments we consider $m=m^{\prime}$ (the same situation suggested by the automatic process), i.e., the relevance and fragmentation features are always of the same length.

Notice that as we increase the size of $m=m^{\prime}$ the performance of the proposed method declines, this means that, considering the relevance and fragmentation features of portions of reused text with length equal or greater than 6 is not very useful for the proposed method in the METER corpus. Furthermore, it is also possible to observe that the size for the context window $v$ that allows to obtain higher values for the $F_{1}$ measure, is in most of the cases $v=19$, and particularly when $m$ and $m^{\prime}$ are equal to $5, F_{1}=0.688$.

As final conclusion, we can claim that proposed heuristic for the automatic definition of the parameter values made a very good approximation of the optimal values, allowing to obtain a result that is only $0.72 \%$ below the best performance.

\subsubsection{Additional experiments on the PSA corpus}

Similarly to the previous section, figure 7 depicts the performance of our proposed algorithm when the three main parameters are manually fixed.

Notice that, similar to the METER corpus, for the PSA considering portions of reused text with length equal or greater than 6 results in a bad performance. Consequently, most of the higher $F_{1}$ scores are obtained when $m$ and $m^{\prime}$ are equal to 5 .

An important difference that we observed when performing these experiments, is that apparently the best context window size was $v=25$, allowing to obtain a $F_{1}$ score of 0.639 . However, remember that the automatic process for defining the parameter values suggested that for the PSA corpus $m=5, m^{\prime}=1$ and $v=19$, allowing us to obtain $F_{1}=0.701$. In order to clarify this behaviour, we performed the experiments showed in figure 8. Such experiments consisted in fixing the values of the context window in 19 and 25, and also fixing $m=5$; the only variation across experiments is the value of $m^{\prime}$.

As it is possible to observe, our automatically defined values $(\mathbf{\Delta})$ for the parameter values are in fact the configuration that allows to obtain the best performance.

\section{Conclusions and Future Work}

In this paper we have proposed a new method for detecting document plagiarism. Its main contribution focuses on the identification of similar and -possible- 


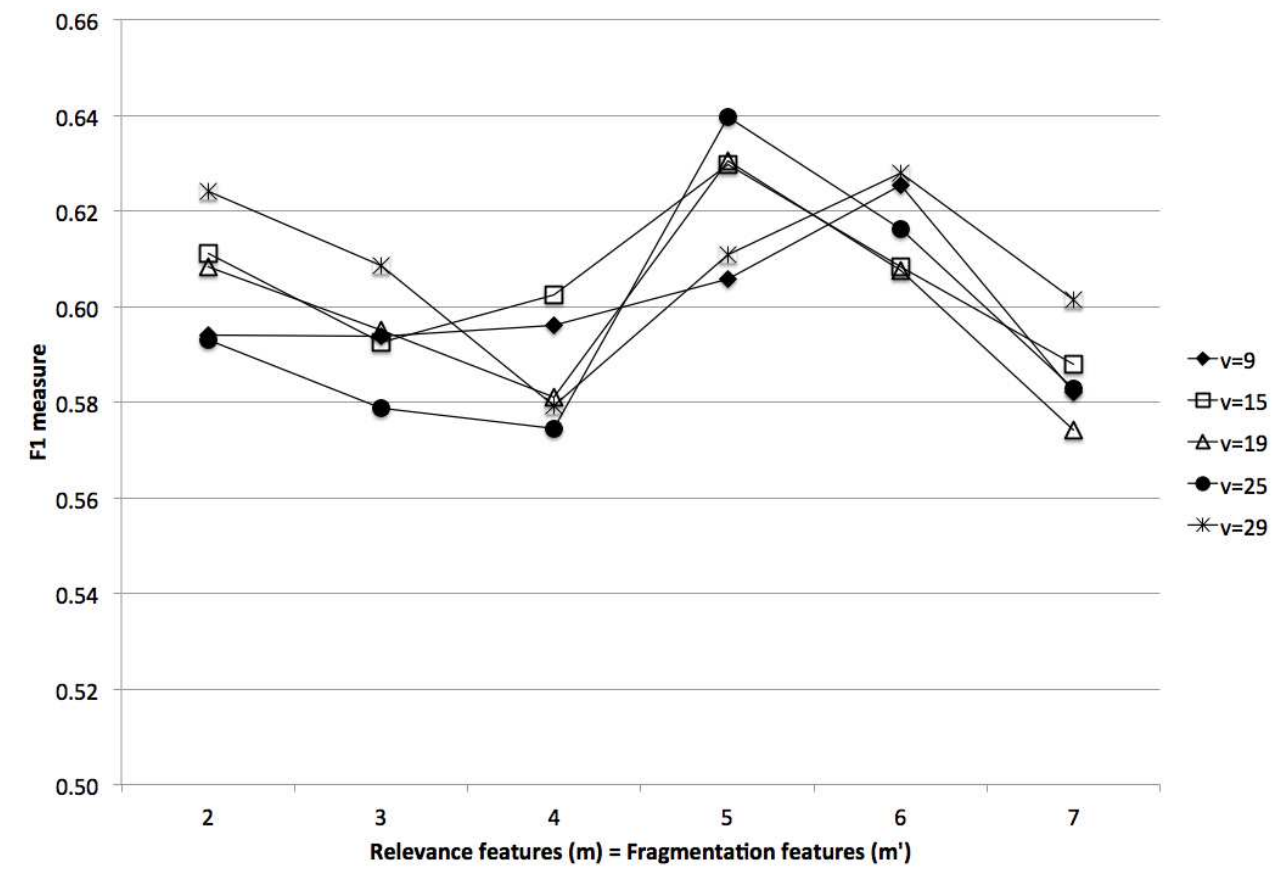

Figure 7: Behaviour of the proposed method when varying the size of the context window $v$, and the maximum length of the relevance and fragmentation features $m$ and $m^{\prime}$ for the PSA corpus.

reused word strings between a original and a suspicious document that are not necessary an exact copy. This method, called the Rewriting Index, assigns a weight to each word from the suspicious document in order to describe its degree of membership to a portion of plagiarized text. This way, it is able to discover text that has suffered from some modifications such as word elimination, insertion, and transposition, allowing to perform a partial matching between documents.

Another important contribution of this paper is the proposal of a richer representation of the portions of reused text. This new representation helps the classification algorithms to better discriminate between plagiarized and non-plagiarized documents by including features that describe not only the number of reused text portions but also their relevance and fragmentation. Additionally, we have proposed a simple methodology that allows our proposed method for automatically select the best configuration of its three main parameter values.

Experimental results on the METER and PSA corpora are encouraging since they showed the appropriateness of the proposed method for the task at hand. Particularly, they outperformed the accuracy results from current methods by $5.2 \%$ and $7.1 \%$ on the METER and PSA corpora, respectively. 


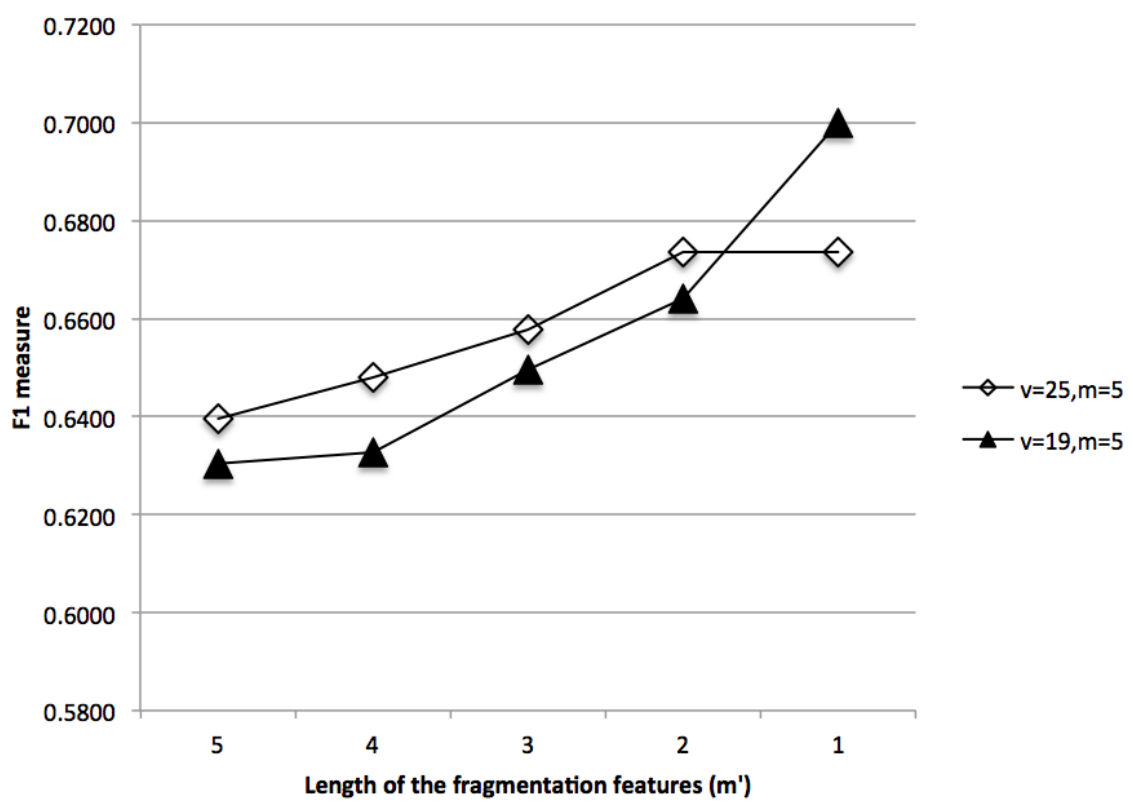

Figure 8: Behaviour of the proposed method when varying the length of the fragmentation features $m^{\prime}$ for the PSA corpus.

As future work we plan to improve the Rewriting Index method by considering synonyms and applying some morphological normalizations. In addition, we plan to explore the use of the ReI feature as a document similarity measure in other related tasks such as document classification and document clustering.

\section{References}

[1] Barrón-Cedeño A., Basile C., Esposti M. D., and Rosso P. (2010) Word Length n-grams for Text Re-Use Detection. In 11th International Conference on Intelligent Text Processing and Computational Linguistics (CICLing '10). LNCS Vol. 6008, Springer Verlag, pp. 687-699.

[2] Barrón-Cedeño A., Eiselt A., and Rosso P. (2009) Monolingual Text Similarity Measures: A Comparison of Models over Wikipedia Articles Revisions. In Proceedings of ICON-2009: 7th International Conference on Natural Language Processing. pp. 29-38. Hyderabad, India.

[3] Barrón-Cedeño A., and Rosso P. (2009) On Automatic Plagiarism Detection Based on $n$-grams Comparison. In Proceedings of the 31th European Con- 
ference on IR Research on Advances in Information Retrieval (ECIR) LNCS Vol. 5478, Springer-Verlag, pp. 696-700.basil Berlin, Heidelberg.

[4] Basile C., Benedetto D., Caglioti E., Cristadoro G., and Degli Esposti M. (2009) A Plagiarism Detection Procedure in Three Steps: Selection, Matches and "Squares". In Proceedings of the SEPLN 2009 Workshop on Uncovering Plagiarism, Authorship and Social Software Misuse (PAN 2009), CEUR-WS Vol. 502. Donostia-San Sebastian, Spain.

[5] Butler M. (2010). Journals Step up Plagiarism Policing. In Nature Vol 466 Num. 7303. July 2010.

[6] Chien-Ying C., Jen-Yuan Y., and Hao-Ren K. (2010) Plagiarism Detection Using ROUGE and WordNet. In Journal of Computing. Vol. 2, Num 3, pp. 34-44.

[7] Chong B. M., Specia L. and Mitkov R. (2010) Using Natural Language Processing for Automatic Detection of Plagiarism. In Proceedings of the 4th International Plagiarism Conference. Newcastle-upon-Tyne, UK.

[8] Clough P. (2003) Old a new Challenges in Automatic Plagiarism Detection. In National Plagiarism Advisory Service pp. 391-407

[9] Clough P., Gaizauskas R., Piao S., and Wilks Y. (2002) METER: Measuring Text Reuse. In Proceedings of the 40th Annual Meeting of the Association for Computational Linguistics (ACL) Philadelphia.

[10] Clough P., and Stevenson M. (2010) Developing A Corpus of Plagiarised Short Answers. In Language Resources and Evaluation: Special Issue on Plagiarism and Authorship Analysis. Volume 45(1), pp. 5-24.

[11] Engles S., Lakshmanan V., Craig M. (2007) Plagiarism Detection Using Feature-Based Neural Networks. In Proceedings of the 38th SIGCSE Technical Symposium on Computer Science Education (SIGCSE '07). pp. 34-38.

[12] Gaizauskas R., Foster J., Wilks Y., Arundel J., Clough P., and Piao S. (2001) The METER Corpus: A Corpus for Analysing Journalistic Text Reuse. In Proceedings of Corpus Linguistics 2001. pp. 214-223. Lancaster, UK. 
[13] Grozea G. C., and Popescu M. (2010) Who's the thief? Automatic Detection of the Direction of Plagiarism. In 11th International Conference on Intelligent Text Processing and Computational Linguistics (CICLing '10). LNCS Vol. 6008. Springer-Verlag pp. 700-710.

[14] Grozea G. C., and Popescu M. (2011) The Encoplot Similarity Measure for Automatic Detection of Plagiarism. In Notebook for PAN at CLEF 2011.

[15] HaCohen-Kerner Y., Tayeb A., and Ben-Dror. (2010) Detection of Simple Plagiarism in Computer Science Papers. In Proceedings of the 23rd International Conference on Computational Linguistics (Coling 2010). pp. 421-429. Beijing, China.

[16] Hartrumpf A. S., Brúck T., and Eichhorn C. (2010) Semantic Duplicate Identification with Parsing and Machine Learning. In Eleventh International Conference on Text, Speech and Dialogue (TSD 2010) LNAI Vol. 6231, Springer-Verlag. pp. 84-92. Brno, Czech Republic.

[17] Hoad T. C., and Zobel J. (2003) Methods for Identifying Versioned and Plagiarized Documents. In Journal of the American Society for Information Science and Technology Vol. 54, Num. 3, pp. 203-215

[18] Nawab R. M. A., Stevenson M., and Clough P. (2011) External Plagiarism Detection using Information Retrieval and Sequence Alignment. In Notebook for PAN at CLEF 2011. Amsterdam, Netherlands.

[19] Oberreuter G., L’Huillier G., Ríos S. A., and Velásquez J. D. (2011) Approaches for Intrinsic and External Plagiarism Detection. In Notebook for PAN at CLEF 2011. Amsterdam, Netherlands.

[20] Potthast M., Stein B., Eiselt A., Barrón-Cedeño A., and Rosso P. (2010) An Evaluation Framework for Plagiarism Detection. In Proceedings of the 23th International Conference on Computational Linguistics (Coling 2010). Beijing, China.

[21] Rao S., Gupta P, Singhal K., and Majumder P. (2011) External \& Intrinsic Plagiarism Detection: VSM \& Discourse Markers based Approach. In Notebook for PAN at CLEF 2011. Amsterdam, Netherlands. 
[22] Seo F. J., and Croft W. B. (2008) Local Text Reuse Detection. In Proceedings of the 31st annual international ACM SIGIR conference on Research and development in Information Retrieval (SIGIR'08).

[23] Si A., Leong H. V., and Lau R. W. H. (1997) CHECK: A Document Plagiarism Detection System. In Proceedings of the 1997 ACM Symposium an Applied Computing. pp. 70-77. San Jose CA, USA.

[24] Shivakumar D. N., and García-Molina Héctor (1995) SCAM: A Copy Detection Mechanism for Digital Documents. In Proceedings of the Second Annual Conference on the Theory and Practice of Digital Libraries. Austin, Texas.

[25] Stein B., and Eissen S. M. (2006) Near Similarity Search and Plagiarism Analysis. In Society 2006. as a selected paper from the 29th Annual Conference of the German Classification Society (GfKl). pp. 430-437.

[26] Suárez P., González J. C., and Villena-Román J.(2011) A Plagiarism Detector for Intrinsic Plagiarism. In Notebook for PAN at CLEF 2011.

[27] Zechner M., Muhr M., Kern R., and Granitzer M. (2009) External and Intrinsic Plagiarism Detection using Vector Space Models. In Proceedings of the SEPLN 2009 Workshop on Uncovering Plagiarism, Authorship and Social Software Misuse (PAN 2009). CEUR-WS Vol. 502. Donostia-San Sebastian, Spain. 